\title{
Plasma fluctuations at the flanks of the Earth's magnetosheath at ion kinetic scales
}

\author{
Liudmila Rakhmanova, Maria Riazantseva, and Georgy Zastenker \\ Space Research Institute of the Russian Academy of Sciences, 84/32 Profsoyuznaya Street, \\ Moscow, 117997, Russian Federation \\ Correspondence to: Liudmila Rakhmanova (rakhlud@gmail.com)
}

Received: 11 May 2016 - Revised: 7 September 2016 - Accepted: 17 October 2016 - Published: 16 November 2016

\begin{abstract}
We present a statistical study of the magnetosheath plasma fluctuation spectra at a high-frequency range (with frequencies from 0.01 to $10 \mathrm{~Hz}$ ). Variations of ion flux value and its direction are considered. The direction of ion flux is characterized by a polar angle - the deviation of the ion flux vector from the Sun-Earth line. We consider 290 Fourier's spectra that can be described by two power laws with a break, i.e., a change of slope. The ion flux fluctuation spectra are shown to have breaks at higher frequencies compared to the polar angle spectra. We compare the frequency of the break with the gyrostructure frequency for a number of cases. We show the polar angle break frequency to usually be smaller than the gyrostructure frequency. The dependencies of spectrum parameters such as the slopes and the break frequency on plasma parameters are also considered.
\end{abstract}

Keywords. Interplanetary physics (solar wind plasma) magnetospheric physics (magnetosheath) - space plasma physics (turbulence)

\section{Introduction}

Space plasma is often in a turbulent state and can serve as a natural laboratory for turbulence exploration. Nowadays a number of spacecraft scanning the solar wind (SW) and the Earth's magnetosheath (MSH) provide large data sets of in situ measurements for the exploration of turbulence in these two regions. Solar wind turbulence has been studied for the past several decades. The results of the investigation are summarized in Alexandrova et al. (2013). Thus, the solar wind turbulence cascade was well discussed at the MHD scales and around the ion characteristic scales (e.g., Markovskii et al., 2008; Riazantseva et al., 2015; Šafránková et al., 2013a,
$2015,2016)$ as well as between the ion and electron scales (e.g., Chen et al., 2012, 2013a, b; Sahraoui et al., 2013).

The magnetosheath turbulence is less well studied. Unlike the solar wind turbulence, it evolves between two boundaries - the magnetopause and the bow shock. The main feature of the magnetosheath is the ion temperature anisotropy that results from the presence of the boundaries and is also a source of free energy that leads to wave generation. A variety of wave modes and instabilities observed in the MSH is described in Lacombe et Belmont (1995) and Schwartz et al. (1996). Spectra of the magnetic fluctuations in the MSH in a large frequency range were discussed by a number of researchers (e.g., Anderson et al., 1994; Czaykowska et al., 2001; Sahraoui et al., 2006, 2013; Alexandrova, 2008; Alexandrova et al., 2008; Huang et al., 2014). These spectra exhibit the presence of several scales, as it was shown for solar wind spectra. As far as we are aware, spectra of plasma (i.e., ion flux or ion density) fluctuations in the magnetosheath are described for frequencies as small as $1 \mathrm{~Hz}$ (e.g., Shevyrev et al., 2003) for the lack of direct plasma measurements with better temporal resolution. A statistical analysis of the ion flux fluctuation spectra together with a comparison between the solar wind and magnetosheath spectra was discussed in Riazantseva et al. (2016). The present study continues the study of Riazantseva et al. (2016). We deal with the enlarged statistics and consider fluctuations of both ion flux value and its direction. We present a statistical analysis of spectral properties of the magnetosheath plasma fluctuations with frequencies up to $10 \mathrm{~Hz}$. We consider a set of the spectrum indexes and break frequencies and compare them to those obtained previously for the solar wind plasma fluctuations as well as magnetosheath magnetic field fluctuations. Also, we compare the frequency of the break to various 
plasma and magnetic field parameters in order to uncover the processes that are responsible for the change in slope.

Note that ion cyclotron waves and mirror waves are supposed to be dominant in the MSH at the frequencies explored in the present study (e.g., Alexandrova et al., 2004; Sahraoui et al., 2003; Anderson et al., 1994). However, the identification of wave modes requires magnetic field measurements unavailable on Spektr-R. For this reason, we do not distinguish wave modes affecting the plasma fluctuation spectra in the present paper.

\section{Observations}

Our study is based on data of the BMSW (fast solar wind monitor) instrument (Zastenker et al., 2013; Šafránková et al., 2013b) on board the Spektr-R spacecraft. Though Spektr$\mathrm{R}$ was designed for astrophysical purposes, its orbit (apogee $\sim 52 R_{\mathrm{E}}$, perigee $\sim 0.1 R_{\mathrm{E}}$, duration $\sim 8$ days) is convenient for monitoring the SW and MSH parameters. The BMSW instrument located on the solar panel of the spacecraft is angled nearly directly at the Sun (within $15^{\circ}$ ) and provides continuous measurements of the solar wind plasma parameters. The instrument is equipped with the DSS probe determining the precise direction of the Sun. BMSW consists of six Faraday cups: three of them face sunward and provide the ion flux value measurements with $31 \mathrm{~ms}$ resolution and the other three cups are inclined by $20^{\circ}$ and used for determination of the flux direction.

An example of the BMSW measurements in the MSH is shown in Fig. 1. The spacecraft location for the analyzed case is $\{-9.6,-18,18\} R_{\mathrm{E}}$ in the GSE coordinate system. Figure 1 demonstrates a typical ion flux value and polar angle measurements at the MSH flank. The polar angle $-\theta-$ is defined as the angle between the ion flux vector and the instrument axis $X_{\mathrm{BMSW}}$. The angle between $X_{\mathrm{BMSW}}$ axis and the Sun-Earth line $\left(X_{\mathrm{GSE}}\right)$ usually varies within the limits of $15^{\circ}$. This angle is measured by the DSS probe. Thus, one has to use a further parameter determined with some errors in order to recalculate the polar angle to the GSE system. Such recalculation results in additional noise arising at high frequencies, which we are interested in. For this reason in the present study, we deal with the polar angle in the BMSW coordinate system. As we concentrate on the fluctuations of the polar angle and its fluctuation spectra, such an approach seems to be reasonable.

A comparison of the ion flux value and polar angle measurements with the help of the BMSW instrument in the SW was discussed by Zastenker et al. (2015). The authors showed these two quantities to be non-correlated with each other in the majority of cases. Both quantities demonstrated rapid variations with the level of polar angle variations being higher (in a statistical sense) than the level of the flux value variations. We suppose that the ion flux value fluctuations represent fluctuations of ion number density (see Pitňa

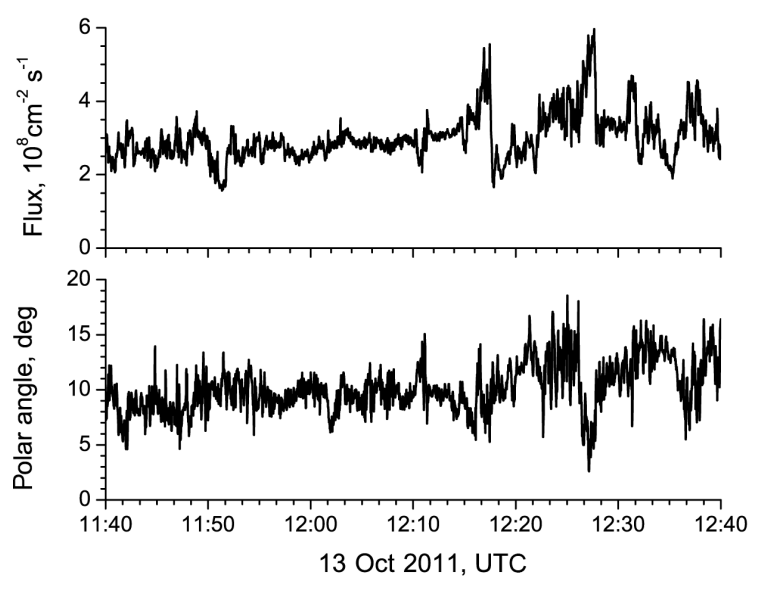

Figure 1. An example of the magnetosheath ion flux value (top panel) and polar angle (bottom panel) measurements by BMSW during period 11:40-12:40 on 13 October 2011.

et al., 2016, for a comparison between the density and ion flux fluctuation spectra by using BMSW data), whereas the polar angle fluctuations represent predominantly the plasma bulk velocity variations.

In the example presented in Fig. 1, the polar angle seems to vary stronger than the flux value. However, to compare levels of fluctuations themselves, one should deal with normalized quantities. Before calculation of the spectra, we normalized data to the mean value for the interval. Examples of the spectra are presented in Fig. 2. The spectra are calculated at $\sim 17 \mathrm{~min}$ interval ( $\sim 32000$ points). Black and red curves show the spectra of the normalized ion flux value and polar angle fluctuations, respectively. One can see the power spectral density (PSD) of both normalized quantities to be of the same order of magnitude. We emphasize the fact that instrumental noise for the ion flux value measurements become significant at frequencies $\sim 10 \mathrm{~Hz}$ and higher (see Šafránková et al., 2013b). For this reason the frequencies above $10 \mathrm{~Hz}$ are not shown in Fig. 2. Since there are three Faraday cups that take part in the polar angle determination, the polar angle measurement errors increase. Moreover, the measurement errors of the inclined cups themselves are higher than those of the non-inclined cups due to lower values of the incoming flux. For these reasons the instrumental noise for the polar angle measurements usually occurs at frequencies lower than that for the flux value. In the case shown in Fig. 2, a flattening of the spectra of the polar angle fluctuations at frequencies above $4 \mathrm{~Hz}$ (denoted with the vertical dotted line) is due to the instrumental noise.

The spectra of the ion flux as well as of the polar angle fluctuations exhibit two power laws separated with breaks. Below the break (at MHD scales), the slope of the ion flux fluctuation spectra is $S_{1}^{\text {flux }}=-1.78$, which is consistent with Kolmogorov's theory. The polar angle fluctuation spectra are flatter at MHD scales, $S_{1}^{\text {angle }}=-1.36$. At higher frequencies 


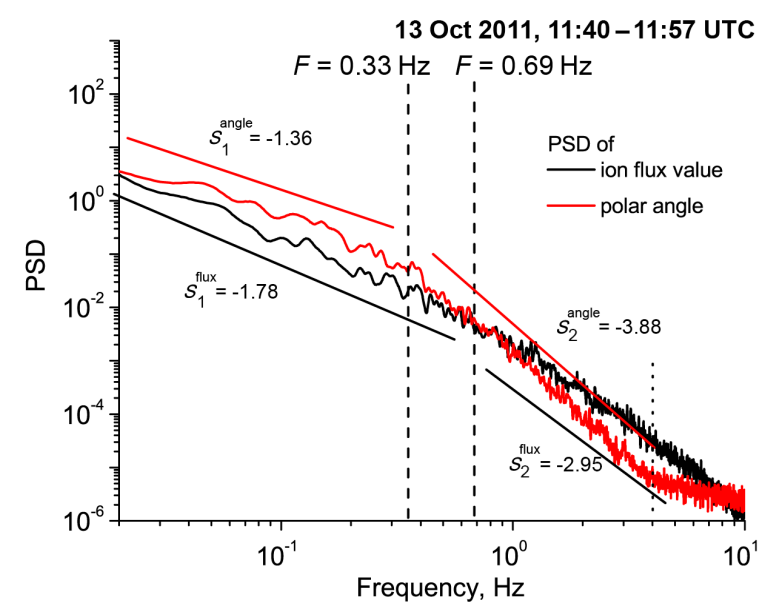

Figure 2. Example of normalized ion flux (black curves) and polar angle (red curves) fluctuation spectra. Vertical dashed lines denote frequencies of the breaks. The dotted line denotes the frequency of the noise beginning at the polar angle fluctuations. Straight lines represent linear approximations of every part of the spectra. The values of the spectral slopes are given next to each line.

(kinetic scales) the spectra become steeper: $S_{2}^{\text {flux }}=-2.95$ and $S_{2}^{\text {angle }}=-3.88$. The spectral breaks occur at frequencies 0.69 and $0.33 \mathrm{~Hz}$ for the ion flux and polar angle fluctuations, respectively.

\section{Statistics}

Altogether, we managed to find $\sim 170 \mathrm{~h}$ of BMSW measurements in the MSH during 2011-2013. Spektr-R traversed the MSH at the flanks. The spacecraft trajectories are displayed in Fig. 3. Dotted lines refer to an average model location of the magnetopause and the bow shock. Note that the positions of the boundaries are different for each crossing due to the different conditions in the upstream solar wind. For this reason some parts of the trajectories do not lie in the MSH according to Fig. 3. However, every crossing was verified manually with the help of plasma parameter measurements and energetic spectrograms to ensure that the spacecraft does locate in the MSH. Our statistics cover a broad range of plasma conditions: plasma density values cover a range from 3 to $70 \mathrm{~cm}^{-3}$ with a mean value of $22 \mathrm{~cm}^{-3}$ and bulk velocity ranges from 230 to $500 \mathrm{~km} \mathrm{~s}^{-1}$ with a mean value of $330 \mathrm{~km} \mathrm{~s}^{-1}$. High values of the bulk velocity (up to $500 \mathrm{~km} \mathrm{~s}^{-1}$ ) are quite rare and can be caused by the spacecraft location at the MSH flank and near the magnetotail.

For further study, we use $\sim 17 \mathrm{~min}$ intervals (32768 data points) overlapped by half of their duration. This criterion was chosen to enable a reliable determination of spectral slopes together with nearly constant plasma parameters within the intervals. The whole statistical set includes 1227 intervals. We eliminate the intervals with the average

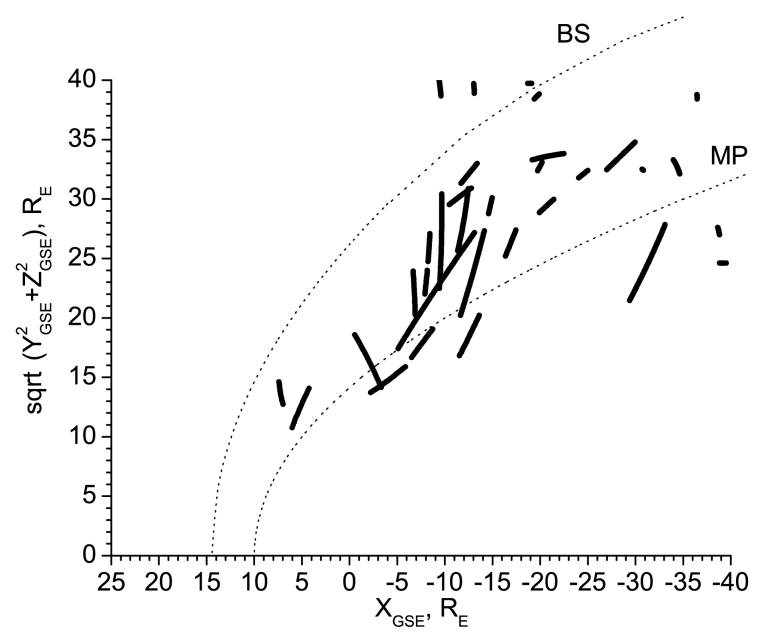

Figure 3. Spektr-R positions during the analyzed intervals. Dotted lines denote the average location of the magnetopause (MP) and the bow shock (BS).

polar angle value $\theta>20^{\circ}$ due to the increasing errors of the parameter calculations (see Gagua et al., 2009, for a detailed description of the method for the flux and polar angle determination). This procedure reduces our statistical set to 671 intervals. We calculate Fourier spectra of the ion flux value and polar angle fluctuations in the range of $0.02-16 \mathrm{~Hz}$ for the whole statistical set. Due to the difference in the frequency of the noise appearance, the approximation procedure is limited by $10 \mathrm{~Hz}$ for the ion flux value fluctuation spectra and by $4 \mathrm{~Hz}$ for the polar angle spectra. We observe spectra of various types: with two power laws, similar to those shown in Fig. 2 and by Riazantseva et al. (2016), with a flattening in a break vicinity, like those shown by Chen et al. (2013b) and Šafránková et al. (2013a, 2015), and with peaks in a vicinity of the break, similar to those shown by Alexandrova et al. (2006) and Alexandrova (2008). In this paper, we focus on the spectra with two clear power laws divided with the break at ion scales amounting to $43 \%$ of cases, i.e., 290 spectra.

We compare PSD for both quantities in the MSH. We calculate PSD on two scales: $2 \pm 0.1$ and $0.02 \pm 0.001 \mathrm{~Hz}$, to estimate a level of the high-frequency (HF) and low-frequency (LF) fluctuations. The two ranges are generally associated with kinetic and MHD scales, respectively (statistics of the break frequency values are discussed further in the text). The PSD histograms together with corresponding Gauss distributions for both quantities at both scales are presented in Fig. 4. The distributions show that (i) at kinetic scales both flux value and polar angle fluctuation levels are by $4-5$ orders of magnitude lower than at MHD scales and (ii) power density of the ion flux value fluctuations is slightly higher than that of the polar angle fluctuations though the difference lies within the standard deviations.

Figure 5a-d show the spectral indexes for all intervals. The slope values below $(S 1)$ and above $(S 2)$ the spectral break are 


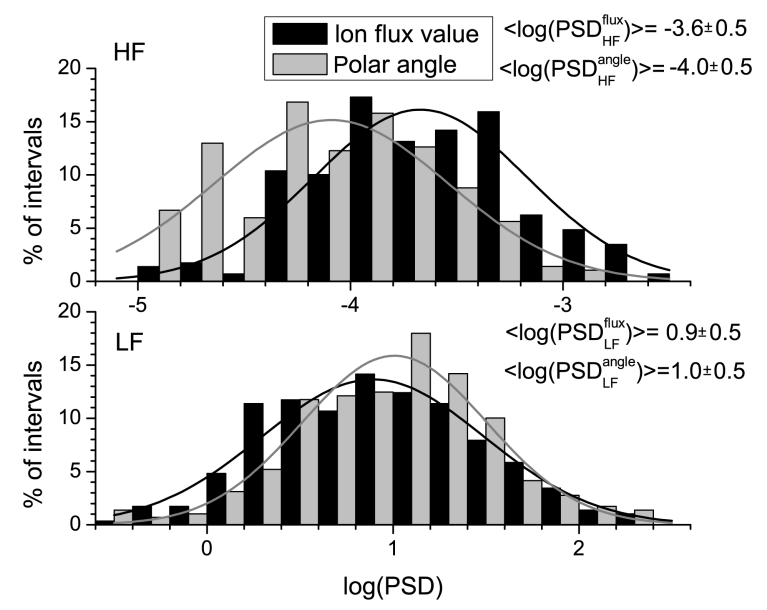

Figure 4. Histograms of PSD values for high-frequency (HF, top panel) and low-frequency (LF, bottom panel) fluctuations of ion flux value (black columns) and polar angle (grey columns) in the MSH. Gauss fits and their parameters for each distribution are shown.

shown in panels a and $b$, respectively; the break frequency $F_{\text {break }}$ is shown in panel c. Black histograms (hereinafter) refer to the spectra of the ion flux value fluctuations and grey histograms to the spectra of the polar angle fluctuations. The Gaussian fits of the distributions together with their parameters (mean values and standard deviations) are also shown in each panel. For both quantities the slopes below the spectral break cover a range $[-2.5,-1.2]$ with mean values of $-1.8 \pm 0.2$ and $-1.7 \pm 0.2$, respectively. This result is similar to the result of Riazantseva et al. (2016) for the ion flux fluctuation spectra in the MSH. Taking into account standard deviations, these values correspond to $-5 / 3$ predicted by Kolmogorov's theory. This result is also consistent with Alexandrova et al. (2008), who reported the value of $-5 / 3$ for the spectral slope of the magnetic field fluctuations in the flank MSH below the break.

For the ion flux value, the slopes above the spectral break also show a broad distribution peaked at $-2.9 \pm 0.3$, as shown by Riazantseva et al. (2016) for the ion flux fluctuation spectra in the MSH. This value is consistent with the results of the statistical study of the MSH magnetic spectra by Huang et al. (2014) and the results of a number of case studies by Alexandrova el al. (2008). However, the polar angle spectra are usually slightly steeper in the dissipation range with the slopes ranging from -4.2 to -2.2 with a mean value of $-3.4 \pm 0.6$. All the distributions are nearly Gaussian.

The spectral break of the ion flux fluctuations ranges from 0.08 to 3.8. However, the number of spectra with $F_{\text {break }}^{\text {flux }}>1.8 \mathrm{~Hz}$ is small, $<5 \%$. The distribution may be described by two Gaussian fits with $\left\langle F_{\text {break_1 }}^{\text {flux }}>=0.45 \pm 0.17 \mathrm{~Hz}\right.$ and $<F_{\text {break } 2 \text { flux }}^{\text {fla }}>=0.9 \pm 0.4 \mathrm{~Hz}$. The two-peak approximation was chosen since the same distribution in the SW exhibits two clear peaks (discussed further in this section).
In the SW, the break frequency of the ion flux fluctuation spectra is significantly higher $-1.9 \pm 0.8$ (Riazantseva et al., 2016). For the polar angle fluctuations the distribution of the break frequencies is more confined - from 0.07 to $1.04 \mathrm{~Hz}-$ and can be described with a Gauss peak with a mean value of $0.36 \pm 0.16 \mathrm{~Hz}$.

To make sure that the difference between the break frequencies of different parameters takes place, we present the distribution of the frequency ratio $-F_{\text {break }}^{\text {flux }} / F_{\text {break }}^{\text {angle }}-$ calculated for each spectrum (shown in Fig. 5d). One can see that usually the spectral break of the ion flux value fluctuations in the MSH occurs at frequencies $2.1 \pm 1.0$ times higher than the break of the polar angle fluctuation spectra. Thus, in the MSH the fluctuation spectra of the ion flux value and polar angle exhibit significant differences that are statistically verified.

The slopes of the ion flux fluctuation spectra were shown to be nearly the same in the SW and MSH in both frequency ranges under study (Riazantseva et al., 2016). Figure 5 demonstrates that the slopes of the ion flux value and polar angle fluctuation spectra in the MSH are roughly equal. Thus, one can suppose the ion flux value and polar angle spectral slopes to also be roughly equal in the SW. This hypothesis was verified. The mean values of the polar angle spectral slopes are $S_{1}=-1.7 \pm 0.2$ and $S_{2}=-3.0 \pm 0.5$ in the frequency range below and above the break, respectively. Taking into account standard deviations, these values are consistent with the values of the slopes of the ion flux fluctuation spectra in the SW (shown by Riazantseva et al. (2016) to be $S_{1}=-1.6 \pm 0.2$ and $S_{2}=-2.9 \pm 0.5$ ) and with the values of the slopes of the polar angle fluctuation spectra in the MSH (presented above).

Since we have observed the difference in the break frequencies of the flux value and polar angle fluctuations in the MSH, a comparison between these quantities in the SW is required. Figure 5e presents histograms of the break frequencies of the flux value and polar angle fluctuation spectra in the SW. Figure 5f represents the distribution of the frequency ratio calculated for each spectra in the SW. The distribution of the break frequencies of the ion flux fluctuation spectra show a clear two-peak structure with peaks at $0.8 \pm 0.3$ and $2.0 \pm 0.7$. In the MSH the break frequencies are smaller and the two peaks are not as clearly observable. This structure of the distribution indicates the presence of two different types of spectra with the break frequencies differing by 2-2.5 times. This difference may be due to different dissipation mechanisms. However, this issue is not within the scope of the present paper and worth further study in the future. The polar angle fluctuation spectra in the SW have breaks at frequencies from 0.08 to 1.95 with a mean value of $0.5 \pm 0.2 \mathrm{~Hz}$. Panel $\mathrm{f}$ in Fig. 5 indicates that in the SW the flux value fluctuation spectra have the breaks at frequencies $2.5 \pm 1.0$ times higher than the polar angle fluctuation spectra. Šafránková et al. (2013) have 
(a)
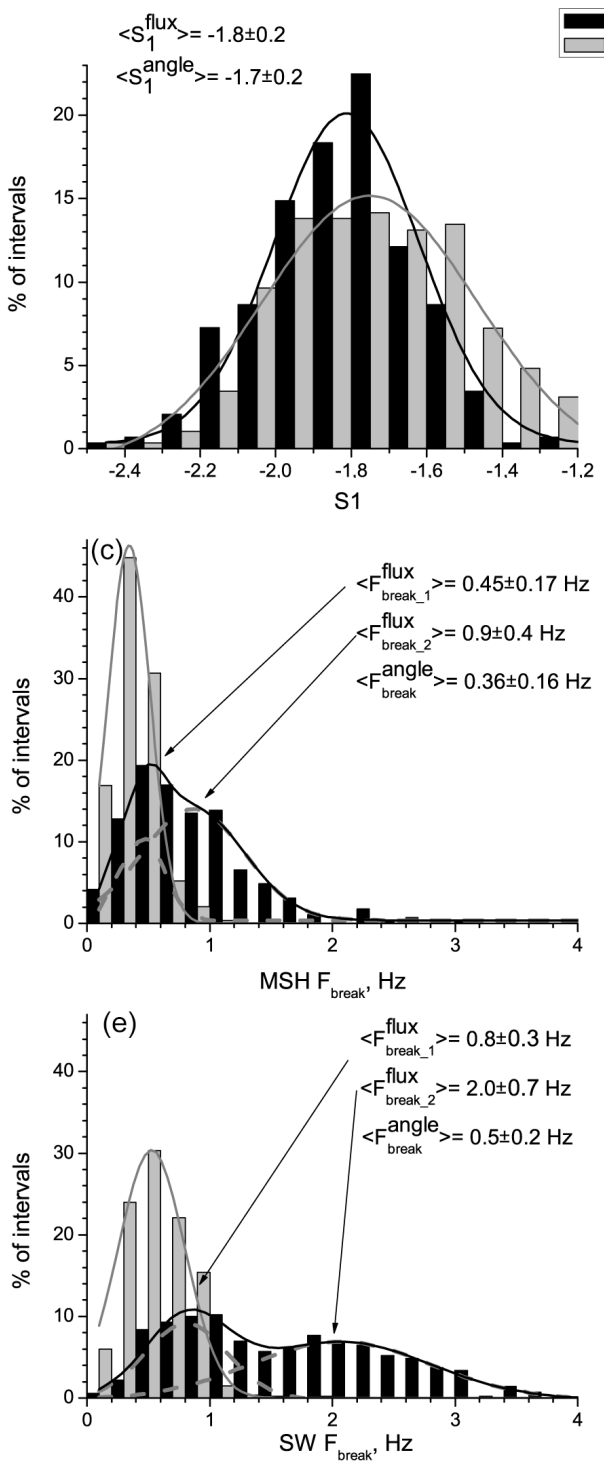

(b)

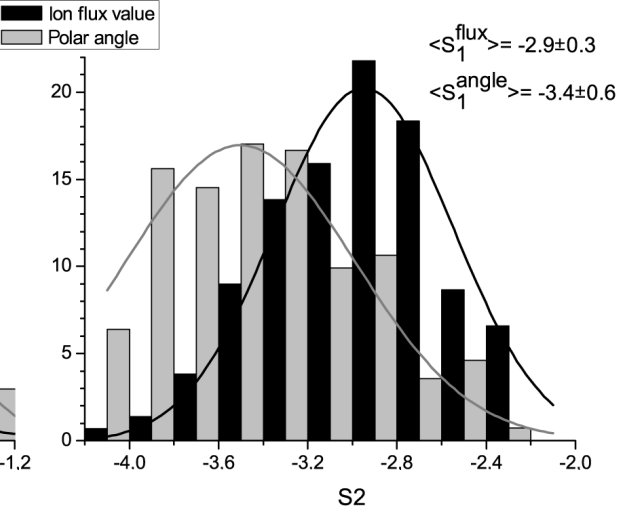

(d)
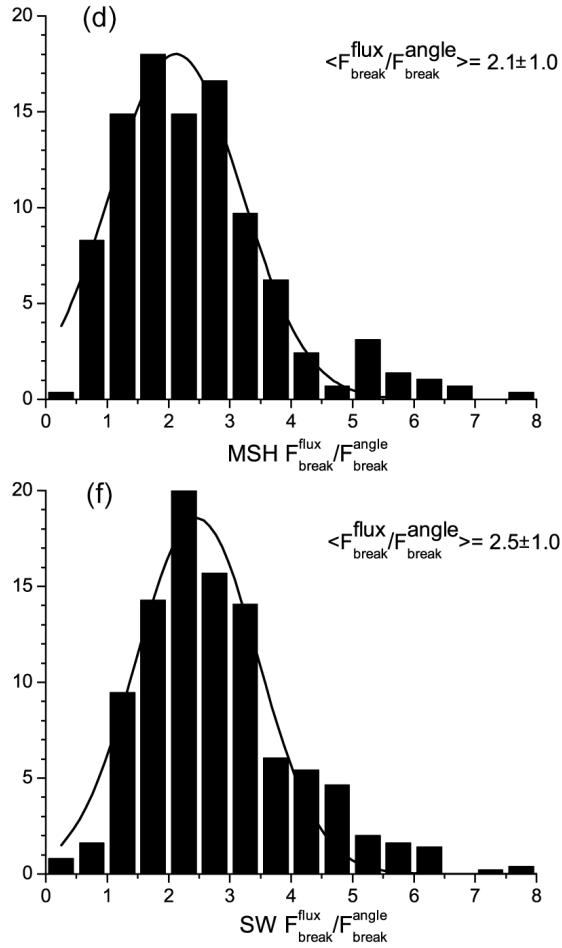

Figure 5. Histograms of the slopes (a) below the break and (b) above the break for the ion flux value (black) and polar angle (grey) fluctuation spectra in the MSH. Frequency of the break for the ion flux value and polar angle spectra (c) in the MSH and (e) in the SW. Histograms of the ratio between the break frequency of the flux value and polar angle fluctuation spectra (d) in the MSH and (f) in the SW. Corresponding Gauss distributions together with their parameters (mean values and standard deviations) are shown for every histogram.

estimated the values of the spectral indexes for the SW ion density and bulk velocity fluctuations. The values reported are $S_{1}^{N}=-1.45, S_{2}^{N}=-3.37$ and $F_{\text {break }}^{N}=1.59 \mathrm{~Hz}$ for density and $S_{1}^{V}=-1.59, S_{2}^{V}=-2.9$ and $F_{\text {break }}^{V}=0.38 \mathrm{~Hz}$ for velocity, where $S_{1}, S_{2}$ and $F_{\text {break }}$ are the slopes below the break, above the break and the break frequency, respectively, and $N$ and $V$ refer to the density and velocity spectra, respectively. The values of the spectral indexes obtained by Šafránková et al. (2013) for the density and velocity fluctuations are consistent with those for the flux value reported by Riazantseva et al. (2016) and for the polar angle presented in this study, respectively. Moreover, Šafránková et al. (2016) showed the break frequency of the SW density fluctuation spectra to always be higher than those of the bulk velocity fluctuation spectra. That is the case for the ion flux and polar angle fluctuation spectra. This fact confirms our suggestion that the flux value fluctuations represent the fluctuations of density whereas the polar angle fluctuations represent the variations of bulk velocity.

Thus, the spectra of the ion flux value fluctuations usually have the break at frequencies 2-2.5 times higher than those of the polar angle fluctuations in the MSH as well as in the SW. Ion flux fluctuations seem to have spectra of two types 


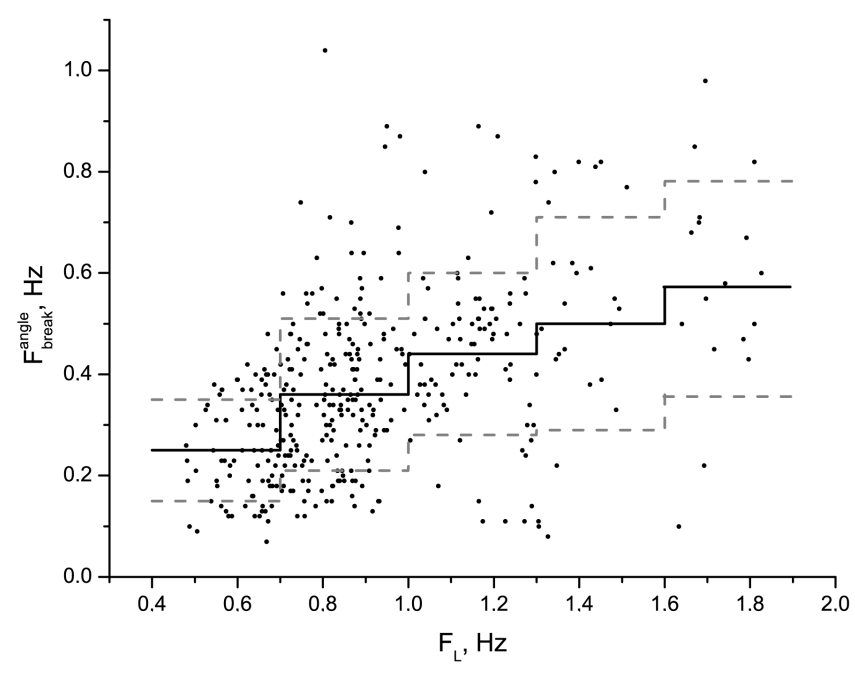

Figure 6. Break frequency of the polar angle spectra as a function of the inertial length frequency $\left(F_{\mathrm{L}}\right)$. Solid black steps refer to mean values for five ranges of $F_{\mathrm{L}}$. Dashed grey steps represent standard deviation from the means.

with the break frequencies differing by a factor of $2-2.5$ in both regions.

To find out if there are some factors influencing the spectral indexes we calculate a correlation coefficient between the spectral indexes and plasma parameters - density, bulk velocity and the inertial length frequency. The latter was chosen following Šafránková et al. (2015). The inertial length frequency is defined as $F_{\mathrm{L}}=V_{\text {bulk }} / 2 \pi L$, where $L=c / \omega_{\mathrm{p}}$ with $\omega_{\mathrm{p}}$ being the proton plasma frequency and $V_{\text {bulk }}$ being bulk velocity. Significant correlation $-R=0.48$ - occurs only between the break frequency of the polar angle fluctuation spectra and the inertial length frequency. Figure 6 demonstrates the corresponding dependence. Since there is a large spread of data points, we do not fit them with a curve. Instead, we present median values calculated in five equidistant ranges of the inertial length frequency.

\subsection{Break frequency of the plasma fluctuations as a function of gyrostructure frequency}

Another characteristic scale supposedly related to the break between MHD and kinetic scales is the thermal gyroradius, $R_{\mathrm{T}}$ (e.g., Galtier, 2006; Schekochihin et al., 2009; Šafránková et al., 2015). $R_{\mathrm{T}}$ is defined as $R_{\mathrm{T}}=V_{\mathrm{th}} / \omega_{C}$ where $V_{\text {th }}$ is the ion thermal speed. To find out if the scaling of plasma fluctuations is related to the thermal gyroradius, we compare the break frequencies of the plasma fluctuation spectra with the gyrostructure frequency, $F_{\mathrm{G}}$, defined as $F_{\mathrm{G}}=V_{\mathrm{bulk}} / 2 \pi R_{\mathrm{T}}$. Since magnetic field measurements are not available on Spektr-R, we have to use magnetic field data from other spacecraft to calculate $F_{\mathrm{G}}$. We managed to find one interval when Themis-B (Angelopoulos, 2008) measurements in the MSH were available and

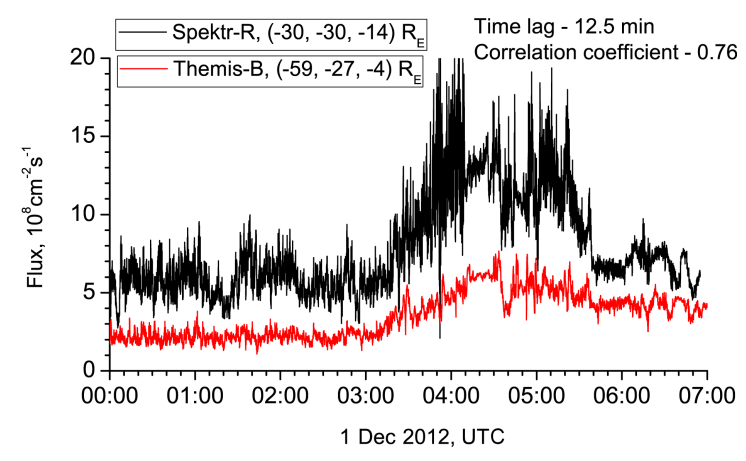

Figure 7. Ion flux measured by Spektr-R (black line) and by Themis-B (red line) on 1 December 2012. Themis-B data are shifted back by 12.5 min to match Spektr-R data.

Spektr-R and Themis-B's mutual location fit to data tracing. Themis-B plasma measurements by the ESA instrument (McFadden et al., 2008) and magnetic field measurements by the FGM instrument (Auster et al., 2008) were taken from http://cdaweb.gsfc.nasa.gov/. The interval is 23:50 06:55 UTC on 30 November-1 December 2012. Spektr-R is located at $\{-30,-30,-14\} R_{\mathrm{E}}$ in the GSE system, Themis$\mathrm{B}$ is located downstream from Spektr-R at $\{-59,-30,-4\}$ $R_{\mathrm{E}}$. The ion flux measurements from both spacecraft are presented in Fig. 7.

Themis-B data are shifted by -12.5 min that corresponds to the plasma propagation time. The correlation coefficient between two time series is 0.76 .

We calculate the frequency spectra with a 1 min step for this case. Figure 8 presents the break frequencies for the ion flux value (black triangles) and for the polar angle (red dots) fluctuations vs. the gyrostructure frequency. Šafránková et al. (2015) reported the high correlation between the break frequency of the ion density fluctuation spectra and the gyrostructure frequency. Šafránková et al. (2016) showed that the break frequency of the density as well as the bulk velocity fluctuations is always lower than the gyrostructure frequency. According to Fig. 8 the break frequency of the ion flux fluctuations does not exhibit any dependence on the gyrostructure frequency, though these frequencies are of the same order of magnitude. The polar angle fluctuation spectra have the break at frequencies lower than the gyrostructure frequency. Similar dependence was shown for the bulk velocity spectra.

\section{Conclusions}

We have presented a statistical study of the frequency spectral indexes for the ion flux value and polar angle highfrequency fluctuations in the Earth's magnetosheath. Our results can be summarized as follows:

- Below the break, the spectral slopes of both quantities' fluctuations correspond to those predicted by Kolmogorov's theory, $-5 / 3$. This result is consistent with 


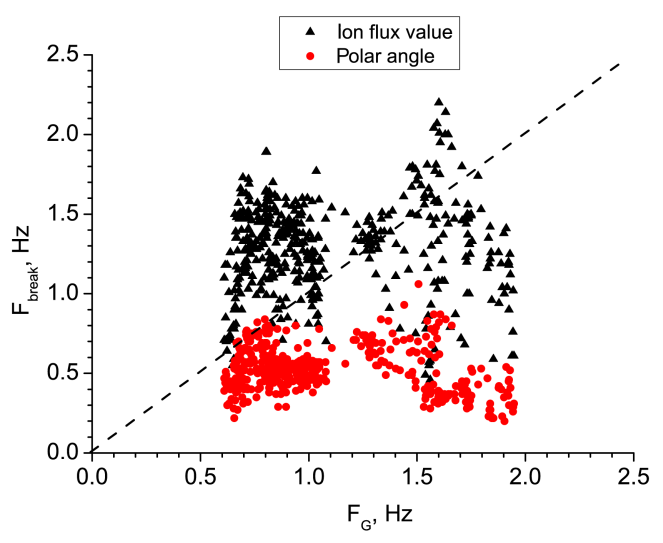

Figure 8. The break frequencies of the ion flux (black triangles) and polar angle (red dots) fluctuation spectra vs. the gyrostructure frequency $\left(F_{\mathrm{G}}\right)$. Dashed line denotes $F_{\text {break }}=F_{\mathrm{G}}$.

previous studies performed for the MSH magnetic field fluctuations (e.g., Alexandrova et al., 2008).

- Above the break the ion flux value fluctuation spectra have slopes of $-2.9 \pm 0.3$. This result is consistent with the results of Huang et al. (2014) and Alexandrova et al. (2008) obtained for the MSH magnetic field. The polar angle fluctuation spectra in this range are usually steeper than those of the ion flux value with a slope of $-3.4 \pm 0.6$.

- For both frequency ranges and for both quantities, the values of the spectral slopes in the MSH match the values of those in the SW (taking into account standard deviations).

- Spectra of the plasma fluctuations in the MSH have breaks at smaller frequencies compared to those in the $\mathrm{SW}$. In the MSH, the spectra of the ion flux value fluctuations exhibit two different groups with break frequencies of $0.45 \pm 0.17$ and $0.9 \pm 0.4 \mathrm{~Hz}$. In the SW there are also two different types of ion flux fluctuation spectra: with the break at $0.8 \pm 0.3$ and $2.0 \pm 0.7 \mathrm{~Hz}$. The presence of the ion flux fluctuation spectra of two different types may be caused by different processes forming the dissipation range. However, this topic should be studied more accurately.

- The polar angle fluctuation spectra have the break at frequencies 2-2.5 times smaller than the ion flux value fluctuation spectra: at frequencies $0.36 \pm 0.16 \mathrm{~Hz}$ in the $\mathrm{MSH}$ and at frequencies $0.5 \pm 0.2 \mathrm{~Hz}$ in the SW.

- The correlation between the spectral indexes and ambient plasma parameters seems to be small. The only significant dependence ( $R=0.48$ ) occurs for the break frequency of the polar angle fluctuations vs. the inertial length frequency. We did not find any dependence of other spectral indexes on plasma parameters.
Šafránková et al. (2015) have reported high correlation ( $R=0.73$ ) between the break frequency of the SW density fluctuation spectra and the gyrostructure frequency. The case study shown in the present paper does not reveal any relation between the break frequency of the MSH flux value fluctuation spectra and the gyrostructure frequency. The polar angle fluctuation spectra have the break at frequencies lower than the gyrostructure frequency. The latter result is consistent with the results of Šafránková et al. (2016) obtained for the bulk velocity spectra. This consistence shows the close relation between the polar angle and bulk velocity fluctuations.

\section{Data availability}

The data set is available on request from the Plasma-F team, at the Experiment BMSW web page: http://aurora.troja.mff. cuni.cz/spektr-r/project/ and at the Plasma-F web page: http: //plasma-f.cosmos.ru/.

Acknowledgements. The reported study was funded by RFBR according to the research project nos. 16-32-00818, 16-02-00669 and 16-02-00125, and by program no. 7 of Fundamental Research of the Russian Academy of Sciences.

The topical editor, C. Owen, thanks the two anonymous referees for help in evaluating this paper.

\section{References}

Alexandrova, O.: Solar wind vs magnetosheath turbulence and Alfvén vortices, Nonlin. Processes Geophys., 15, 95-108, doi:10.5194/npg-15-95-2008, 2008.

Alexandrova, O., Mangeney, A., Maksimovic, M., Lacombe, C., Cornilleau-Wehrlin, N., Lucek, E. A., Décréau, P. M. E., Bosqued, J.-M., Travnicek, P., and Fazakerley, A. N.: Cluster observations of finite amplitude Alfvén waves and small-scale magnetic filaments downstream of a quasi-perpendicular shock, J. Geophys. Res., 109, A05207, doi:10.1029/2003JA010056, 2004.

Alexandrova, O., Mangeney, A., Maksimovic, M., CornilleauWehrlin, N., Bosqued, J.-M., and André, M.: Alfvén vortex filaments observed in magnetosheath downstream of a quasiperpendicular bow shock, J. Geophys. Res., 111, A12208, doi:10.1029/2006JA011934, 2006.

Alexandrova, O., Lacombe, C., and Mangeney, A.: Spectra and anisotropy of magnetic fluctuations in the Earth's magnetosheath: Cluster observations, Ann. Geophys., 26, 3585-3596, doi:10.5194/angeo-26-3585-2008, 2008.

Alexandrova, O., Chen, C. H. K., Sorriso-Valvo, L., Horbury, T. S., and Bale, S. D.: Solar Wind Turbulence and the Role of Ion Instabilities, Space Sci. Rev., 178, 101-139, doi:10.1007/s11214 013-0004-8, 2013.

Anderson, J., Fuselier, S., Gary, S., and Denton, R.: Magnetic spectral signatures in the Earth's magnetosheath and plasma depletion layer, J. Geophys. Res., 99, 5877-5891, 1994. 
Angelopoulos, V.: The THEMIS mission, Space Sci. Rev., 141, 534, doi:10.1007/s11214-008-9336-1, 2008.

Auster, H. U., Glassmeier, K. H., Magnes, W., Aydogar, O., Baumjohann, W., Constantinescu, D., Fischer, D., Fornacon, K. H., Georgescu, E., Harvey, P., Hillenmaier, O., Kroth, R., Ludlam, M., Narita, Y., Nakamura, R., Okrafka, K., Plaschke, F., Richter, I., Schwarzl, H., Stoll, B., Valavanoglou, A., and Wiedemann, M.: The THEMIS fluxgate magnetometer, Space Sci. Rev., 141, 235-264, doi:10.1007/s11214-008-9365-9, 2008.

Chen, C. H. K., Salem, C. S., Bonnell, J. W., Mozer, F. S., and Bale, S. D.: Density Fluctuation Spectrum of Solar Wind Turbulence between Ion and Electron Scales, Phys. Rev. Lett., 109, 035001, doi:10.1103/PhysRevLett.109.035001, 2012.

Chen, C. H. K., Boldyrev, S., Xia, Q., and Perez, J. C.: Nature of Subproton Scale Turbulence in the Solar Wind, Phys. Rev. Lett., 110, 225002, doi:10.1103/PhysRevLett.110.225002, $2013 \mathrm{a}$.

Chen, C. H. K., Howes, G. G., Bonnell, J. W., Mozer, F. S., Klein, K. G., and Bale, S. D.: Kinetic Scale Density Fluctuations in the Solar Wind, AIP Conf. Proc., 1539, 143-146, doi:10.1063/1.4811008, 2013b.

Czaykowska, A., Bauer, T. M., Treumann, R. A., and Baumjohann, W.: Magnetic field fluctuations across the Earth's bow shock, Ann. Geophys., 19, 275-287, doi:10.5194/angeo-19-275-2001, 2001.

Galtier, S.: Wave turbulence in incompressible Hall magnetohydrodynamics, J. Plasma Phys., 72, 721, doi:10.1017/S0022377806004521, 2006.

Gagua, I. T., Gagua, T. I., and Zastenker, G. N.: Determination of a solar wind ion flux value and direction using a set of integral Faraday cups for the fast monitor of solar wind, in: WDS'09 Proceedings of Contributed Papers, Part II: Physics of Plasmas and Ionized Media, 2-5 June 2009, Prague, Czech Republic, 22-29, 2009.

Huang, S. Y., Sahraoui, F., Deng, X. H., He, J. S., Yuan, Z. G., Zhou, M., Pang, Y., and Fu, H. S.: Kinetic turbulence in the terrestrial magnetosheath: Cluster observations, Astrophys. J. Letters, 789, L28, 6 pp., doi:10.1088/2041-8205/789/2/L28, 2014.

Lacombe, C. and Belmont, G.: Waves in the Earth's magnetosheath: Observations and interpretations, Adv. Space Res., 15, 329-340, 1995.

Markovskii, S. A., Vasquez, B. J., and Smith, C. W.: Statistical analysis of the high-frequency spectral break of the solar wind turbulence at $1 \mathrm{AU}$, Astrophys. J., 675, 1576-1583, doi:10.1086/527431, 2008.

McFadden, J. P., Carlson, C. W., Larson, D., Ludlam, M., Abiad, R., Elliott, B., Turin, P., Marckwordt, M., and Angelopoulos, V.: The THEMIS ESA plasma instrument and in-flight calibration, Space Sci. Rev., 141, 277-302, doi:10.1007/s11214-008-9440-2, 2008.

Pitňa, A., Šafránková, J., Němeček, Z., Goncharov, O., Němec, F., Přech, L., Chen, C. H. K., and Zastenker, G. N.: Density fluctuations upstream and downstream of interplanetary shocks, Astrophys. J., 819, 41, 9 pp., doi:10.3847/0004-637X/819/1/41, 2016.

Riazantseva, M. O., Budaev, V. P., Zelenyi, L. M., Zastenker, G. N., Pavlos, G. P., Safrankova, J., Nemecek, Z., Prech, L., and Nemec, F.: Dynamic properties of small scale solar wind plasma fluctuations, Philos. T. R. Soc. A, 373, 20140146, doi:10.1098/rsta.2014.0146, 2015.
Riazantseva, M. O., Budaev, V. P., Rakhmanova, L. S., Zastenker, G. N., Šafránková, J., Němeček, Z., and Přech, L.: Comparison of properties of small scale ion flux fluctuations in flank magnetosheath and in solar wind, Adv. Space Res., 58, 166-174, doi:10.1016/j.asr.2015.12.022, 2016.

Šafránková, J., Němeček, Z., Přech, L., and Zastenker, G. N.: Ion Kinetic Scale in the Solar Wind Observed, Phys. Rev. Lett., 110, 025004, doi:10.1103/PhysRevLett. 110.025004, 2013a.

Šafránková, J., Němeček, Z., Přech, L., Zastenker, G. N., Cěrmák, I., Chesalin, L., Komárek, A., Vaverka, J., Beránek, M., Pavlu, J., Gavrilova, E., Karimov, B., and Leibov, A.: Fast Solar Wind Monitor (BMSW): Description and First Results, Space Sci. Rev., 175, 165-182, $2013 b$.

Šafránková, J., Němeček, Z., Němec, F., Přech, L., Pitňa, A., Chen, C. H. K., and Zastenker, G. N.: Solar wind density spectra around the ion spectral break, Astrophys. J., 803, 107, 7 pp., doi:10.1088/0004-637X/803/2/107, 2015.

Šafránková, J., Němeček, Z., Němec, F., Přech, L., Chen, C. H. K., and Zastenker, G. N.: Power spectral density of fluctuations of bulk and thermal speeds in the solar wind, Astrophys. J., 825, 121, 8 pp., doi:10.3847/0004-637X/825/2/121, 2016.

Sahraoui, F., Belmont, G., Rezeau, L., Cornilleau-Wehrlin, N., Pinçon, J. L., and Balogh, A.: Anisotropic Turbulent Spectra in the Terrestrial Magnetosheath as Seen by the Cluster Spacecraft, Phys. Rev. Lett., 96, 075002 , doi:10.1103/PhysRevLett.96.075002, 2006.

Sahraoui, F., Huang, S. Y., Belmont, G., Goldstein, M. L., Rétino, A., Robert, P., and De Patoul, J.: Scaling of the electron dissipation range of solar wind turbulence, Astrophys. J., 777, 15, 11 pp., doi:10.1088/0004-637X/777/1/15, 2013.

Schekochihin, A. A., Cowley, S. C., Dorland, W., Hammett, G. W., Howes, G. G., Quataert, E., and Tatsuno, T.: Astrophysical gyrokinetics: kinetic and fluid turbulent cascades in magnetized weakly collisional plasmas, Astrophys. J., Supplement Series, 182, 310, doi:10.1088/0067-0049/182/1/310, 2009.

Schwartz, S. J., Burgess, D., and Moses, J. J.: Low-frequency waves in the Earth's magnetosheath: present status, Ann. Geophys., 14, 1134-1150, doi:10.1007/s00585-996-1134-z, 1996.

Shevyrev, N., Zastenker, G. N., Nozdrachev, M. N., Němeěek, Z., Šafránková, J., and Richardson, J. D.: High and low frequency large amplitude variations of plasma and magnetic field in the magnetosheath: radial profile and some features, Adv. Space Res. 31, 1389-1394, 2003.

Zastenker, G. N., Šafránková, J., Nvemeveek, Z., Prech, L., Cermak, I., Vaverka, I., Komarek, A., Voita, J., Chesalin, L. S., Karimov, B. T., Agafonov, Yu. N., Borodkova, N. L., Gavrilova, E. A., Gagua, T. I., Gagua, I. T., Dalin, P. A., D'yachkov, A. V., Koloskova, I. V., Leibov, A. V., Semena, N. P., Chernov, V. V., Markov, Ya. I., Ryazanova, E. E., Ryazanrtseva, M. O., Shevyrev, N. N., Chrapchenkov, V. V., Chugunova, O. M., and Yurasov, A. S.: Fast measurements of solar wind parameters by BMSW instrument, Cosmic Res.+, 51, 78-89, doi:10.1134/S0010952513020081, 2013.

Zastenker, G. N., Khrapchenkov, V. V., Koloskova, I. V., Gavrilova, E. A., Ryazanova, E. E., Ryazantseva, M. O., Gagua, T. I., Gagua, I. T., Šafránková, J., Němeček, Z., Přech, L., and Voita, J.: Rapid Variations of the Value and Direction of the Solar Wind Ion Flux, Cosmic Res.+, 53, 59-69, 2015. 